\title{
[18F]-FDG PET/CT for suspected lymphoma relapse in a patient with concomitant pneumococcal pneumonia during COVID-19 outbreak: unexpected SARS-Cov-2 co-infection despite double RT-PCR negativity
}

\author{
Lucia Zanoni ${ }^{1}$ (1) $\cdot$ Cristina Mosconi $^{2} \cdot$ Veronica Cervati $^{1} \cdot$ Margherita Diegoli $^{3} \cdot$ Francesco Monteduro $^{3} \cdot$ Rita Golfieri $^{2}$. \\ Stefano Fanti ${ }^{1}$
}

Received: 30 March 2020 / Accepted: 23 April 2020 / Published online: 19 May 2020

(C) Springer-Verlag GmbH Germany, part of Springer Nature 2020

During COVID-19 outbreak (March 2020), a local 78year-old male patient, with a history of treated nonHodgkin lymphoma, was admitted to the hospital (day 1) with persistent fever, cough, and dyspnea. Thorax high-resolution computed tomography (HRCT) suspected viral lung infection and incidentally detected enlarged axillary and mediastinal lymphadenopathies. Reverse transcriptase-polymerase chain reaction (RTPCR) on pharyngeal swab yielded negative results for SARS-CoV-2 infection, both at patient admission and 2 days after (days 1 and 3). Subsequently (day 5), Streptococcus pneumoniae urinary antigen was found positive; therefore, the patient was immediately referred to high-dose antibiotics administration for pneumonia and to [18F]-FDG PET-CT for suspected lymphoma relapse. The scan (day 6) demonstrated multiple FDG avid lymphadenopathies above and below the diaphragm

This article is part of the Topical Collection on Infection and inflammation

Lucia Zanoni

lucia.zanoni@aosp.bo.it

1 Metropolitan Nuclear Medicine, AOU S.Orsola-Malpighi, Via Albertoni 15, 40138 Bologna (BO), Italy

2 Department of Diagnostic Medicine and Prevention, Radiology Unit (Director Prof. Golfieri), AOU S.Orsola-Malpighi, Bologna, Italy

3 Department of Diagnostic Medicine and Prevention, Radiology Unit-Specialist Section (Director Dott. Monteduro ff), AOU S.Orsola-Malpighi, Bologna, Italy and increased diffuse uptake in the spleen in keeping with NHL progression and suspected bowel involvement. Furthermore, concomitant pneumonia was confirmed due to faint and diffuse uptake within a left inferior lobe consolidation and a single non-FDG-avid peripheral rounded ground-glass opacity (GGO) in the right upper lobe. A follow-up (day 11) HRCT demonstrated GGO small reduction, further extension of left basal consolidation, and new periscissural thickening in the right apex. Then, a third RT-PCR test (day 12) finally revealed COVID-19. Two weeks later, despite hydroxychloroquine and azithromycin, clinical and radiological worsening was documented (day 26) showing extensive interstitial viral involvement throughout both lungs. The patient started tocilizumab and required CPAP ventilation.

Although RT-PCR remains the gold standard for COVID-19 diagnosis, false-negative/delayed results are not uncommon. The routine method for screening, diagnosis, and monitoring is HRCT. [18F]-FDG PET/CT is not specific, and differential diagnosis of lung infections is challenging [1-4].

According to this single experience, we suppose that co-infection with other pathogens (i.e., S. pneumoniae) might influence RT-PCR test accuracy; when clinical and CT features are highly suggestive for suspected COVID-19 pneumonia, precautions in patients management are recommended even in case of first RT-PCR negativities; during COVID-19 pandemic, incidental findings detected by a nuclear medicine physician at lung window CT component of PET/CT studies might be extremely relevant. [5] 


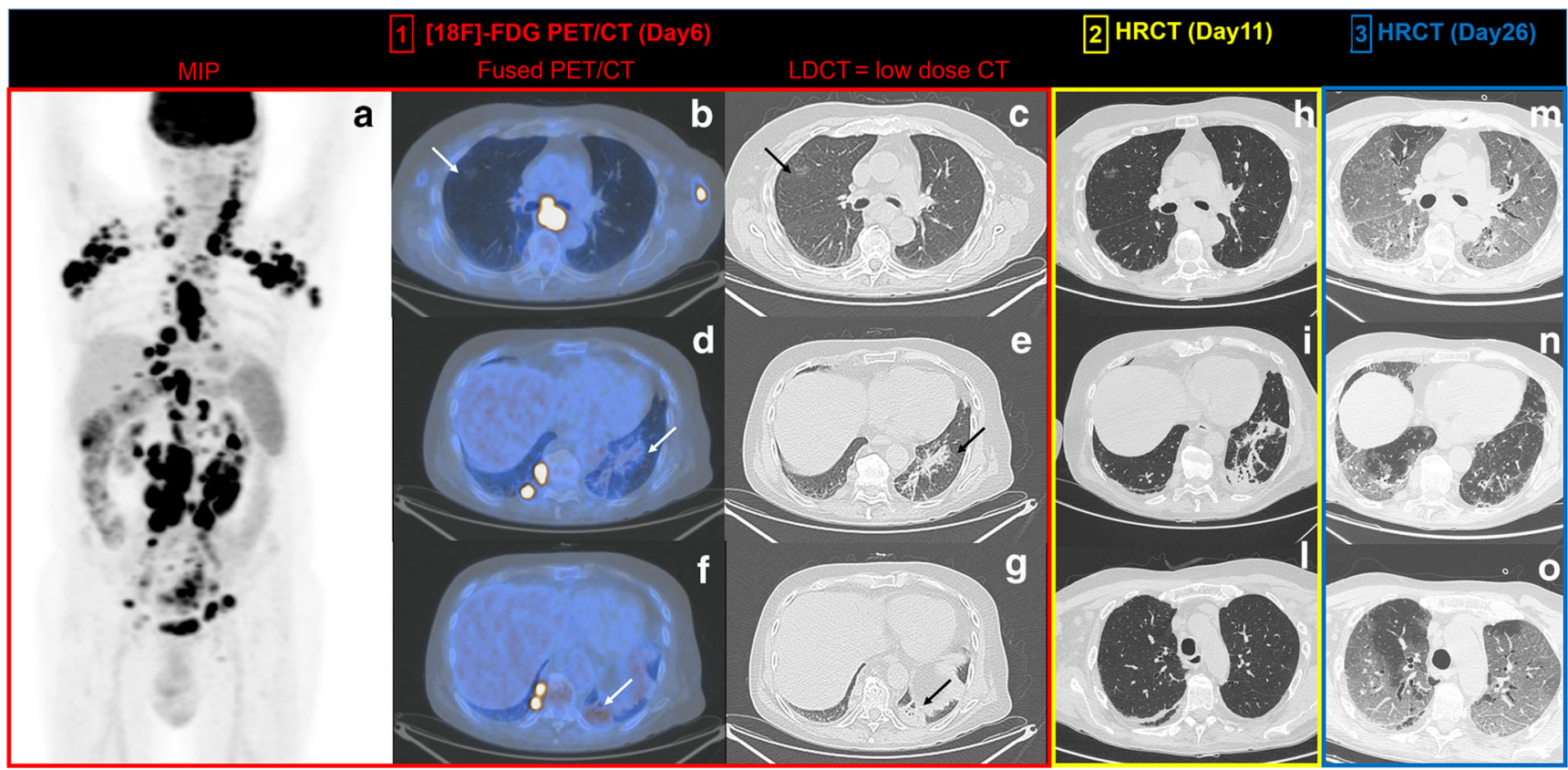

\section{Compliance with ethical standards}

Conflict of interest The authors declare that they have no conflict of interest.

Ethical approval All procedures performed in studies involving human participants were in accordance with the ethical standards of the institutional and/or national research committee and with the 1964 Helsinki declaration and its later amendments or comparable ethical standards.

Informed consent Informed consent was obtained from the participant for all the imaging procedures included. The Local Ethics Committee is not involved in single case report's authorization. Consent from the patient for anonymous publication of the images for scientific purposes was obtained during hospitalization.

\section{References}

1. Deng Y, Lei L, Chen Y, Zhang W. The potential added value of FDG PET/CT for COVID-19 pneumonia. Eur J Nucl Med Mol Imaging. 2020. https://doi.org/10.1007/s00259-020-04767-1.

2. Joob B, Wiwanitkit V. 18F-FDG PET/CT and COVID-19. Eur J Nucl Med Mol Imaging. 2020. https://doi.org/10.1007/s00259-02004762-6.

3. Zou S, Zhu X. FDG PET/CT of COVID-19. Radiology. 2020;6: 200770. https://doi.org/10.1148/radiol.2020200770.

4. Qin C, Liu F, Yen TC, Lan X. (18)F-FDG PET/CT findings of COVID-19: a series of four highly suspected cases. Eur J Nucl Med Mol Imaging. 2020. https://doi.org/10.1007/s00259-02004734-w.

5. Czernin J, Fanti S, Meyer PT, Allen-Auerbach M, Hacker M, Sathekge M, et al. Imaging clinic operations in the times of COVID-19: strategies, precautions and experiences. J Nucl Med. 2020. https://doi.org/10.2967/jnumed.120.245738.

Publisher's note Springer Nature remains neutral with regard to jurisdictional claims in published maps and institutional affiliations. 